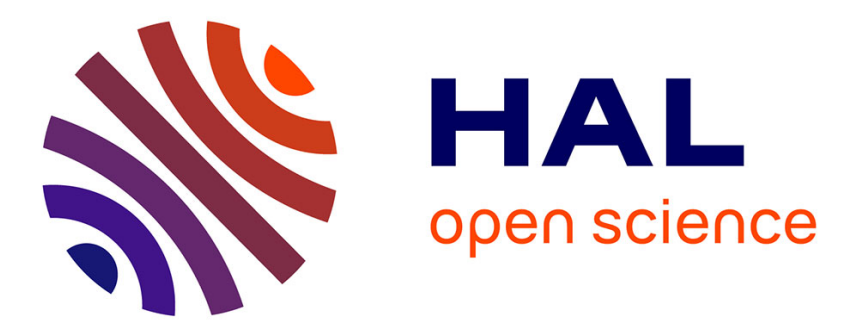

\title{
Grandeur et décadence de la double conservation dans les arènes internationales
}

\author{
David Dumoulin Kervran
}

\section{To cite this version:}

David Dumoulin Kervran. Grandeur et décadence de la double conservation dans les arènes internationales: (appartient au dossier spécial " Environnement et expertise : entre science et politique, quelle légitimité? ", sous la direction de Dalya Guérin). Quaderni, 2007, 64, pp. 23-36. halshs-00490894

\section{HAL Id: halshs-00490894 https://shs.hal.science/halshs-00490894}

Submitted on 10 Jun 2010

HAL is a multi-disciplinary open access archive for the deposit and dissemination of scientific research documents, whether they are published or not. The documents may come from teaching and research institutions in France or abroad, or from public or private research centers.
L'archive ouverte pluridisciplinaire $\mathbf{H A L}$, est destinée au dépôt et à la diffusion de documents scientifiques de niveau recherche, publiés ou non, émanant des établissements d'enseignement et de recherche français ou étrangers, des laboratoires publics ou privés. 


\section{Publié/pour citer:}

- David Dumoulin Kervran, "Grandeur et décadence de la double conservation dans les arènes internationales" (dossier spécial "expertises environnementales sous la direction de Dalya Guérin), Quaderni Université Paris 1, №64, automne 2007, pp. 23-36 (appartient au dossier «Environnement et expertise : entre science et politique, quelle légitimité ?»)

\section{Mots-clés:}

Experts, environnement, double conservation, Amérique latine

\section{Résumé:}

L'influence politique des Experts est ici travaillée par le biais de la construction de la notion d'environnement en Amérique Latine par les Organisations Non Gouvernementales Environnementales (ONGE). L'échange de savoirs entre profanes et savants est l'occasion de montrer en quoi l'élaboration d'une conception juridique de l'environnement est avant tout politique. Dans le cas étudié, l'auteur caractérisera le rôle politique que les experts ONGE ont joué auprès des gouvernements nationaux et des populations locales. Car imposer la définition et la pratique légitime de l'environnement, c'est s'imposer comme le détenteur d'une vision politique de l'environnement.

Il est ici plus précisémént propasé une cartographie des discours de "la double conservation" car les discours qui posent une relation interdépendance entre conservation de la biodiversité et conservation de la diversité culturelle sont en fait tres divers dans leur point de départ, leurs objectifs et degrés d'élaboration.

\section{"Grandeur et décadence de la double conservation dans les arènes internationales"}

David Dumoulin, MCF sociologie IHEAL-UNIVERSITÉ PARIS 3, Doctorat Etudes Politiques de Sciences Po Paris, fin nov. 2003, chercheur associé au CREDAL

Depuis la fin des années 1980, c'est en grande partie dans le sillage de l'internationalisation des politiques écologiques que celle des luttes indigènes a pu rencontrer un certain succès. Certes, l'internationalisation de la cause autochtone avait commencé dès les années 1970, mais le lien entre ces deux thématiques n'était pas souvent affiché en Amérique latine. Aujourd'hui encore, un tel lien semble toujours reposer sur une instrumentalisation réciproque entre organisations indiennes et écologistes, sur un malentendu toujours plus difficile à masquer. Il n'empêche, l'argument écologique est omniprésent dans les discours des leaders internationaux du mouvement autochtone, et même dans la bouche des représentants des organisations locales qui ont fleuri tout au long du continent, au Nord comme au Sud, en Amazonie mais aussi dans les hautes terres. J'appelle “double conservation", l'idée qu'il existe une relation réciproque et nécessaire entre conservation de la biodiversité et défense des populations autochtones (et, par extension, de la diversité culturelle). On en explicitera les principes basiques dans la première partie de ce texte, avant d'exposer ses multiples versions dans la seconde pour comprendre la diversité des intérêts que recouvre ces discours experts ${ }^{1}$. S'interroger sur l'impact international de cette défense de la double conservation permet à la fois de saisir une ressource symbolique importante utilisée dans l'internationalisation de la cause autochtone, et de retracer la trajectoire d'une problématique qui a eu son heure de gloire dans les arènes internationales durant la seconde partie des années 1990. La double conversation pointe non seulement la porosité de l'expertise aux enjeux politiques 
internationaux et locaux du moment, mais aussi l'opportunité de l'échange des discours et pratiques sur l'environnment dans le processusde légitimation et d'institutionalisation des différents protagonistes.

Après avoir mis en cette dynamique institutionnelle, semblant suggérer la création d'un nouveau champ international autour de la double conservation, j'évoquerai la fragilité de cette institutionnalisation et les limitations du rapprochement entre les deux secteurs au début des années 2000. En revanche, le terme de savoirs locaux est bien devenu un de ces mots-clé qu'affectionne la coopération internationale et il a relancé les débats ${ }^{2}$. La dernière partie de ce texte est consacrée à la présentation d'une carte synthétique des arguments de la double conservation que l'on rencontre aujourd'hui. Une boussole est nécessaire pour souligner la diversité des projets politiques qui peuvent se saisir de ce programme.

\section{I - Internationalisation de la question autochtone et arènes}

\section{environnementales : des alliances contre les mega-projets au programme de la double conservation}

Les étapes de l'internationalisation de la problématique autochtone sont à présent bien connues. Il existe en Amérique latine une première dynamique nationale ou continentale : contestation des politiques indigénistes intégrationnistes, émergence puis consolidation des mouvements indigènes indépendants dans les pays, premières organisations régionales comme la Conférence du Cercle Arctique en 1977ou la COICA en 1984. Elle débouche sur la première mobilisation continentale lors de la campagne "500 ans de résistance..." qui non seulement intensifie d'un coup les relations entre organisations indigènes des pays latinoaméricains, mais rapproche également celles-ci de leurs puissantes alter-ego d'Amérique du Nord. La seconde dynamique est celle qui se déroule dans les arènes internationales ellesmêmes et qui est cruciale pour forger, à l'attention des organisations qui émergent dans le monde entier, une plateforme, un langage et des revendications communes. Elle est lancée à partir d'une première conférence sur les ONG et les peuples indigènes en 1977 à l'ONU, et sera suivie, cinq ans plus tard, de la formation du groupe de travail, du projet de déclaration en 1993 et de la Décennie, puis débouchera sur le Forum Permanent de l'ONU en 2002. Dans ces deux dynamiques, les acteurs transnationaux ont un rôle majeur; les réseaux de soutien des anthropologues et de l'Eglise catholique en particulier ont fortement modelé les discours et l'organisation de ce mouvement indigène revendiquant sa différence.

Certains aspects de l'internationalisation de la question indigène restent encore dans l'ombre et demanderaient des études plus précises, en particulier les inégalités entre organisations indigènes elles-mêmes, dans cette course à la légitimation internationale ${ }^{3}$. Mais ce dont il est question ici concerne un autre élément plus fondamental encore dans l'internationalisation de la question indigène : l'utilisation des avancées fulgurantes qu'avait obtenu le thème de la conservation de la nature dès la fin des années 1980. Ce rapprochement est devenu une pièce essentielle de l'attention accordée à la question indigène : il s'appuie sur certaines traditions des populations indigènes et certaines attentes affectives des populations du Nord. Il a pris toute son importance au fur et à mesure qu'il s'avérait que la cause indigène avait le plus grand mal à aboutir par elle même à des résultats définitifs dans les arènes interétatiques. L'étude de ce rapprochement aide donc à comprendre comment peuvent s'enchevêtrer les différents champs de la coopération internationale : les dirigeants indigènes et leurs alliés ont consciemment fait passer leurs efforts d'influence du domaine des droits de l'Homme vers celui de l'environnement. Les gains plus grands et plus rapides qu'ils escomptaient ont d'ailleurs été en partie obtenus. Le champ de la conservation de la nature, qui a canalisé 
durant les années 1990 une attention médiatique et des montants exceptionnels de la coopération internationale, a par ailleurs fait l'objet de tentatives similaires d'utilisation de la part d'autres acteurs luttant en faveur des droits des femmes, des afro-américains, ou même des droits de l'homme.

Quelles ont été les étapes de ce rapprochement entre écologistes et mouvements indigènes ? Avant 1970, les domaines de la conservation de la nature et celui des droits indigènes se construisent de manière totalement séparée; les politiques indigénistes ne conçoivent la spécificité des relations indigènes avec leur environnement que comme un frein au développement et les premiers conservationnistes rêvent de réserves naturelles vides, à l'américaine. Les trois facettes de ce rapprochement vont s'imbriquer de plus en plus intimement au début des années 1990.

- A partir du début des années 1980 et jusqu'à aujourd'hui, se forment des alliances stratégiques écologico-indigènes contre des ennemis communs, contre les méga-projets des Etats et des banques multilatérales en particulier, contre les grands projets pétroliers, miniers, forestiers ou les barrages en terres indigènes. Ceux qui luttent contre "l'ethnocide" et "l'écocide" se découvrent les mêmes intérêts et s'organisent en réseaux transnationaux de militants.

- L'évolution interne des deux champs est marquée par un rapprochement de leurs agendas et acteurs. Durant cette période 1970-1994, le secteur des réserves naturelles commence à prendre en compte le problème indigène et la participation sociale, alors que les réseaux de défense des peuples indigènes utilisent de plus en plus le discours écologiste.

- La création et la diffusion d'un cadre cognitif basé sur l'ethnobotanique ou la réflexion sur "l'éco-développement" tend à démontrer qu'il existe une relation intrinsèque entre "stratégie éco et ethno", entre défense de la diversité naturelle et de la diversité culturelle, et qu'il est donc impératif de créer une politique commune pour leur conservation.

Plus encore que ces projets aux objectifs mixtes surlesquels ont reviendra, il semble bien que ce discours de défense du lien entre diversité culturelle et biologique ait obtenu le succès le plus marquant ${ }^{1}$. Dans les années 1990, la création d'un mouvement transnational en faveur de la diversité culturelle ET naturelle se fait sous l'égide explicite de ce qu'on appelera «le

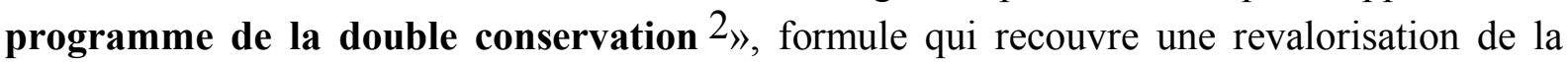
Diversité.

La double conservation a connu une grande diffusion internationale, à partir de l'activité d'un groupe d'experts (ethnobiologistes et anthropologues principalement), qui a su profiter d'alliances avec les organisations indigènes, et établir des contacts avec un secteur de la conservation qui avait besoin de «mettre du social dans sa conservation », pour renforcer sa légitimité et son efficacité. Cette diffusion a été facilitée par un nouvel engouement pour les forêts tropicales, pour la biodiversité; puis pour la nouvelle problématique des droits de propriété intellectuelle, ce qui a permis une propagation vers l'ensemble des financeurs internationaux. Les experts-militants qui défendent ce programme ont été aussi des agents de socialisation dont le rôle a été de traduire les problématiques internationales vers des organisations locales (vocabulaire, "best practices", etc.) qui étaient, jusqu'il y a peu, totalement étrangères à de tels débats surle droit international et l'écologie.

\footnotetext{
1 Ainsi, la publication du Livre Rouge des Peuples Opprimés de Survival International est le pendant du Livre Blanc des espèces animales menacées de l'UICN, ou de la Liste Rouge des Plantes Menacées du World Conservation Monitoring Center, et les trois ouvrages possèdent bien un horizon mondial.

2 Entre la pensée des années 1970 qui en forme les racines et ce nouveau programme, on passe alors de la notion de connaissances paysannes à celle de connaissances autochtones (ethnicisation des discours), d'une insistance sur des stratégies productives alternatives à la conservation de la biodiversité, de la recherche de stratégies nationales à une perception globale des problèmes écologiques et du patrimoine culturel.
} 
La diffusion du programme de la double conservation ne s'est pas seulement appuyée sur un argumentaire déclinant les images de l'indien sage, jardinier et écologiste, sur lesquelles on reviendra. L'élaboration de nouvelles cartes, qui mettent en évidence le recoupement géographique entre zones indigènes et zones de très forte biodiversité, a eu aussi un effet indéniable dans cette diffusion ${ }^{4}$. Des tableaux et schémas mettent en parallèle le nombre d'espèces, classées suivant diverses catégories (mammifères, oiseaux, insectes, plantes cultivées, endémisme), et le nombre de langues existantes (considérées comme le principal élément de la diversité culturelle). Ces études sont en général organisées par pays, échelle à laquelle existent les données collectées, et permettent de créer une nouvelle catégorie : celle de "pays mega-divers". A partir de 1994, le rapide développement des banques de données encyclopédiques créées au niveau mondial sur les connaissances traditionnelles de cette biodiversité constitue un troisième outil réservé aux spécialistes ${ }^{5}$. Alors que se multiplie l'information disponible, tant sur les problèmes juridiques que sur les cas locaux (histoires, acteurs, cartes, problèmes), un "savoir global" émerge bien sur ce nouveau champ.

Ces évolutions seraient-elles le signe que cette double conservation est devenue l'objet d'un nouveau champ de la coopération internationale, à côté de ceux qui lui ont donné naissance, avec un savoir spécifique, des experts, des lignes de financements, des politiques publiques nationales (etc...) ?

\section{II - Projets écologico-indigènes: une croissance institutionnelle}

\section{rapide durant les années 1990.}

A l'intersection entre le champ indigène et le champ de la conservation de la nature, se développe, pendant les années 1990, et avec une intensité particulière à partir de 1995, un nouveau domaine d'activité de la coopération internationale et de l'activisme transnational. Les alliances stratégiques écologico-indigènes et le rapprochement des agendas durant les années 1980 a eu des conséquences institutionnelles avec la multiplication de projets menés en commun qui veulent à la fois défendre les peuples indigènes et conserver la biodiversité. L'exemple le plus frappant est sans doute celui du dispositif des "terres indiennes pour la conservation" qui tend à s'institutionnaliser durant les années 1990 en Amérique latine, à partir du foyer de rayonnement que constitue l'Amazonie brésilienne. Certains acteurs clé doivent être perçus comme des relais dans la diffusion du programme de la double conservation, et comme des canaux de représentation d'intérêts qui étaient auparavant absents. On assiste ainsi à la création de l'entité «Peuples des forêts tropicales », ou à la participation institutionnalisée de lobbies indigènes dans les arènes environnementales internationales. Des normes internationales traitant de ce problème ont aussi été ratifiées par les Etats, et pour ce qui est de la formation d'un personnel spécialisé, l'ethnobiologie est une discipline en pleine croissance durant les années 1990, et, à la même période, l'anthropologie s'est tournée une nouvelle fois vers l'écologie. Il existe donc une expertise internationale de plus en plus large sur ce lien entre diversité culturelle et naturelle.

Quels sont, de manière synthétique, les acteurs non-gouvernementaux spécialisés, les programmes des organisations internationales et les normes qui se sont alors multipliées dans ce nouveau "créneau"?

A partir de 1992 et de la conférence de Rio, les acteurs transnationaux spécialisés sur cette intersection sont de plus en plus nomrbreux. La Société Internationale d'Ethnobiologie (SIE) est explicitement formée en 1988 pour défendre ce lien inextricable entre diversité culturelle et naturelle, et ses experts militants - comme Darell Posey - constituent des foyers d'irradiation de ce programme. Du côté des organisations indigènes, l'Alliance des Peuples 
Indigènes et Tribaux des .Forêts Tropicales (APITFT), formée en février 1992 à Penang (Malaisie), participe très activement au lobbying des organisations internationales, aux côtés de la petite équipe anglaise du "Programme des Peuples Forestiers" du World Rainforest Mouvement. Puis, en 1993, est créé le Réseau mondial Biodiversité des Peuples Indigènes ${ }^{6}$. Proche des milieux écologistes nord-américains très actifs dans ce domaine, l'Amazon Alliance collabore avec la COICA et facilite l'ensemble des contacts des Amazoniens avec les acteurs de Washington, alors que quelques petites ONG comme Native Lands ou Tierra Lingua $^{7}$, ont également un rôle important dans la diffusion du programme de la double conservation.

On trouve ensuite de petites unités spécialisées très actives au sein d'ONG plus grandes et plus anciennes de chacun des deux secteurs. Du côté de la conservation de la nature, les principales sont, par ordre d'importance : celle du WWF avec Gonzalo Oviedo (Peoples \& Conservation Unit) entre 1994 et 2001, le "Peoples, Forest \& Reefs", dirigé par Janis Alcorn au sein du conglomérat "Biodiversity Support Programme »; à l'UICN, l' Unité des Politiques Sociales, qui s'est chargée un moment de la thématique indigène. Dans le champ indigéniste, l'ONG nord-américaine Cultural Survival a œuvré très activement, dès le milieu des années 1980, à la constitution d'un tel lien entre écologistes et organisations indigènes des basses terres en Amérique latine. IWGIA possède quelques spécialistes et quelques publications importantes plus tardives, alors que Survival International est pour sa part en retrait sur cette thématique.

Le "Monde des Etats" (gouvernements et OIG), n'a pas été en reste, mais a réagi plus tard, avec la création d'Unités spéciales à partir de 1994. Marquée par ses premières expériences amazoniennes, la Banque Mondiale, à travers son Département "Développement Environnementalement \& Socialement Durable en Amérique Latine", possède une expertise particulière sur le thème et a produit de nombreuses études pour assurer la diffusion du programme de la double conservation. Sa politique en direction des peuples indigènes a été profondément marquée par sa dépendance envers les projets de conservation de la nature, et la multiplication de ses Plans de Développement Indigène en garde une trace régulière. Les fonds GEF gérés par le département concernent, dans presque tous les pays, des projets explicitement "indigènes", justifiés par le programme de la double conservation. L'Union Européenne a aussi financé des petits programmes de recherche-action autour de la double conservation : pour "donner un visage" à sa ligne budgétaire Forêt Tropicale (DG VIII), elle a financé le projet Avenir des Peuples des Forêts Tropicales (APFT 1994-2000) explicitement chargé d'étayer de manière crtitique le programme de la double conservation et de sensibiliser les bailleurs de fonds.

Le PNUD n'a pas connu d'unité spécifique et ne s'est pas intéressé à la relation entre réserves naturelles et délimitation des terres indiennes. Il a en revanche contribué à diffuser les discussions à propos de la valeur des connaissances indigènes sur la Biodiversité, à partir de 1995, en finançant des consultations et des publications dans plusieurs continents. A l'UNESCO, les experts de la double conservation portent à bout de bras plusieurs petits programmes : ainsi, sur les savoirs autochtones, des ethnobotanistes ont lancé Peoples \& Plants, puis LINKS. Toujours à l'UNESCO, le World Heritage Center permet certaines avancées plus théoriques en diffusant, dans les milieux des experts mondiaux du patrimoine, de nouvelles catégories de conservation: celle de zone préservée pour son importance culturelle ET naturelle (ou mixte), puis, durant les années 1990, celle de paysage culturel et de route culturelle. Il promeut aussi de nouvelles initiatives en faveur des sites sacrés autochtones, préservés en tant que zones de conservation de la biodiversité. Certains gouvernements ont également soutenu dans les arènes internationales le rapprochement entre acteurs écologistes et indigènes, et plus précisément les droits de propriété intellectuelle des 
populations indigènes; il s'agit en particulier du Danemark, de la Hollande, puis de l'Espagne à partir de 1995 .

En plus de cette présence institutionnelle, le second signe de la croissante importance du programme de la double conservation est la multiplication des normes internationales dans ce domaine. Elles sont de statut hétérogène et forment un embryon de régime international qui dépasse les simples références au lien qui unirait les peuples indigènes à leur environnement naturel, telles qu'elles apparaissent dans les premières normes internationales indigènes, comme la convention 169 de l'OIT, l'OP 4.20 de la Banque Mondiale ou même le projet de déclaration de $1^{\prime} \mathrm{ONU}^{8}$. Au premier chef, on pensera à la $\mathrm{CDB}$, avec son célèbre article $8 \mathrm{j}$ (et le 10c sur la conservation in-situ), qui surgit grâce aux réunions de préparation de la $\mathrm{CDB}$, et qui débouche sur une série de principes adoptés directement par les Etats en 2001. Cette avancée dans une arène intergouvernementale importante a été précédée puis accompagnée par l'ouverture d'autres espaces de discussion et par la production d'autres principes internationaux sur la relation entre peuples indigènes et préservation de l'environnement. Ainsi, à partir de l'Agenda 21 publié en 1992, et en particulier de son chapitre 26; les acteurs indigènes et non-indigènes qui défendent la double conservation parviennent à faire adopter des principes allant dans ce sens au sein de la la Commission de Développement durable, puis du Pannel International sur les Forêts, la Convention RAMSAR (zones humides), et la Convention sur la Désertification. Le WWF, puis l'UICN en 1996, diffusent aussi de nouveaux principes d'action censés orienter l'ensemble de leurs projets en faveur d'une reconnaissance d'un partenariat avec les peuples indigènes ${ }^{9}$.

Pour compléter ce corpus de normativité internationale, souvent mobilisé dans les déclarations des experts indigènes, on peut mentionner : la Charte des Peuples Indigènes des Forêts Tropicales, proclamée par l'Alliance du même nom; la Déclaration de Kari Oka qui précède de peu la Conférence de Rio 92, et la Déclaration de Mataatua 96 en Nouvelle Zélande, plus précise sur les savoirs locaux et les droits de propriété intellectuelle. Nouveaux acteurs transnationaux, programmes des OIG et des grandes ONG de chacun des deux champs, nouvelles normes de statut de plus en plus élevé : entre 1994 et 2000, il pouvait sembler qu'un nouveau secteur international était en train de naître sur ce lien entre conservation de la nature et peuples autochtones. Cependant, on sait aujourd'hui qu'il s'agissait en partie d'un phénomène de mode, récurrent dans l'histoire de la coopération internationale, que les deux secteurs ont connu ensuite une sorte de "re-sectorialisation" et que les alliances entre organisations indigènes et écologistes ont fini par révéler les malentendus ou les divergences d'intérêts qui sous-tendent leurs relations.

Malgré les avancées de la décennie 1990, cette institutionnalisation du programme de la double conservation dans les arènes internationales est finalement limitée, et a même régressé si on l'évalue en 2003. Le processus n'a vraiment donné naissance ni à des programmes massifs en faveur de ce lien entre diversité culturelle et biodiversité (sauf ceux de la Banque Mondiale/GEF), ni à une normativité possédant une réelle influence de contrainte (sauf peutêtre le $8 \mathrm{j}$. de la $\mathrm{CDB}$ ). Ce retrait de la marée est principalement dû à quatre nouvelles tendances: les croissantes critiques sur le contenu de la double conservation dans les milieux d'experts, la resectorialisation organisée par les acteurs de chaque champ, la faiblesse du rapprochement au niveau national/local par rapport aux arènes internationales, et la dilution du thème des savoirs locaux dans l'ensemble de l'agenda de la politique internationale. Les limites internes de la double conservation sont l'objet de croissantes critiques, en particulier du côté des experts de la conservation de la nature; à la juste mesure de l'ampleur des projets que ce programme a inspirés durant la décennie 1990. Le programme des experts militants, en particulier lorsqu'il est vulgarisé et transformé en slogan, prête le flanc à une multitude de critiques qui ont freiné sa diffusion. La nécessaire interdisciplinarité utilisée sur le terrain mouvant des relations entre nature et culture peut mener en effet à des généralisations hâtives 
et à des propositions peu responsables en faveur d'une vision statique des communautés ou d'une « métaphysique territoriale » potentiellement excluante. Les experts-militants défendant la double conservation, malgré leur prudence, ont certainement tendance à forcer le trait au sujet du rôle des peuples indigènes dans la conservation de la nature, afin de créer un mouvement politique en leur faveur ${ }^{10}$.

Finalement, il est difficile de cerner les frontières d'un champ de la double conservation, tant la thématique s'est aujourd'hui diffusée vers d'autres secteurs. En tant que discours critique et célébration de la Diversité, la double conservation continue son expansion dans les milieux militants et a vu son audience mondiale revigorée à travers les polémiques sur la problématique des connaissances indigènes sur la biodiversité et leur marchandisation ${ }^{11}$. La double conservation, sans déboucher sur la formation d'un secteur international bien délimité, a connu un grand succès discursif, a favorisé des alliances fécondes et a été intégrée au sein des secteurs déjà constitués : conservation de la nature, lutte contre la pauvreté, valorisation des cultures traditionnelles.

\section{III- Victime de son succès ? Une multitude d'intérêts peut se cacher} derrière le discours de la double conservation

La diffusion du programme de la double conservation, telle qu'on peut l'observer dans la littérature portant sur les populations indiennes et la protection de l'environnement, a entraîné une croissante instrumentalisation. Jusqu'à présent, on a définit le programme de manière minimale : l'idée d'un lien névessaire entre politique de conservation de la nature et politique multiculturaliste, entre diversité biologique et diversité culturelle. En fait il existe une multitude de versions qui peuvent masquer des optiques très différentes derrière cette proclamation générale. La première différence est celle qui sépare une version plus orientée vers la problématique territoriale (entre réserves naturelles et territoires indigènes), et une autre plus centrée sur les connaissances indigènes de la biodiversité (droits de propriété intellectuelle). La première phase, avant 1992, est très largement dominée par la problématique territoriale, avec le développement des alliances opposées à la destruction des forêts et favorables à la démarcation des terres indiennes, celles-ci étant conçues comme zones de conservation de la nature. A partir du milieu des années 1990, le thème des connaissances autochtones prend une importance croissante jusqu'à dominer les débats internationaux sur le lien environnement et peuples autochtones. Il faut noter alors que, pour les dirigeants autchtones, la dimension territoriale prime en général, et que les revendications sur les droits de propriété intellectuelle sont souvent utilisées pour servir un agenda territorial voilé. A l'inverse on peut se demander si les débats sur la propriété des savoirs ne viennent pas parfois occulter ceux qui concernent les luttes autour de la propriété de la terre et des ressources naturelles, ou les politiques extractives.

Comment s'orienter dans cette multitude de positions reliant conservation de la nature et problématique autochtone ? Quelle est la variété et la structure des argumentaires du programme de «la double conservation » ? Un panorama général peut, seul, permettre de comprendre l'ensemble des arguments et leurs différentes combinaisons privilégiées par les différents acteurs. Je propose ci-dessous une sorte de "carte de navigation" qui a été dressée à partir de l'ensemble des lectures et entretiens effectués durant les années de thèse, et qui présente les combinaisons possibles de dix algorithmes. On peut distinguer principalement des versions fortes de la double conservation, deux versions déséquilibrées en faveur de la biodiversité ou de la diversité culturelle, et trois versions instrumentales, dont la finalité est en fait : la lutte contre pauvreté, la valorisation économique, ou la lutte contre le néo-libéralisme. 
Tout d'abord le contexte cognitif dans lequel agissent tous les acteurs de la coopération internationale et du développement local a nettement évolué pendant les années 1990 jusqu'à ce que ceux-ci partagent le plus souvent deux algorithmes :

- 1 - «il existe souvent un recoupement géographique entre les zones habitées par des populations indigènes et les zones de haute biodiversité, de forêts encore bien préservées et de réserves naturelles ».

- 2 - « il est à présent difficile, dans les zones de population indigène, de ne pas travailler directement avec leurs organisations nouvelles sans perdre rapidement sa légitimité politique et morale ».

$\rightarrow$ Beaucoup d'anthropologues et d'ethnobotanistes défendent ce que l'on peut appeler la "version forte de la double conservation" qui s'inscrit dans une critique générale du développement, et qui prend en compte l'avenir de l'humanité toute entière. Leur argument principal est le suivant :

3 - «Il existe un lien inextricable entre diversité biologique et culturelle, entre la mosaïque des éco-systèmes et celle des cultures ; et la conservation de la première ne peut réussir sans la conservation de la seconde, sans la défense des peuples autochtones " En fait, cette affirmation sur l'interdépendance des deux diversités est appuyée par différentes justifications, qui peuvent être avancées suivant des combinaisons différentes. Une version plus proche de la défense de l'idée "d'ethnosciences" : "seuls les peuples indigènes ont une connaissance exceptionnelle de cette biodiversité et de son usage ». Une version spiritualiste : " leur relation d'interdépendance spiritualiste et holiste à la nature, avec leurs sites sacrés et leurs horizons temporels longs, les différencie des stratégies destructives occidentales », et une version insistant plutôt sur leur organisation sociale : " leur cohésion sociale et leur autonomie locale leur permet l'application des normes de bon usage des ressources naturelles $\gg$.

Cette perspective s'appuie sur un argument procédural :

4-« Il existe un cercle vicieux : la destruction environnementale et la perte de la biodiversité mènent à la perte de diversité culturelle (à travers la déstructuration sociale et la pauvreté) et la perte de diversité culturelle à la perte de biodiversité (perte des stratégies diversifiées, du contrôle social sur l'usage du territoire et des ressources, pression croissante sur les ressources). ";

mais aussi sur une justification plus normative :

5- «La diversité culturelle et la diversité naturelle font partie du patrimoine de l'humanité ;

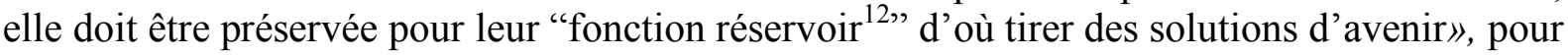
la qualité intrinsèque de la Diversité, ou encore pour leur contribution essentielle à la beauté du monde ».

Ces cinq arguments sont en général présents dans ce qu’on appelle ici la version forte de la double conservation.

Ensuite, il est possible de distinguer deux "versions déséquilibrées" du programme de la double conservation :

$\rightarrow$ Une version, que l'on peut attribuer souvent aux personnes qui travaillent dans le secteur de la conservation de la nature (ou "conservationnistes"), et dont le principal argument, qui s'ajoute aux 1 et 2, est alors :- 6 - « la politique de la conservation in-situ de la biodiversité ne peut fonctionner efficacement sans la participation des populations locales». (affirmation qui 
reste controversée dans le milieu mais représente la version conservationniste de la double conservation).

$\rightarrow$ Une version correspondant plutôt aux discours des leaders indigènes : - 7: « Nous, les indiens, estimons que ces territoires nous appartiennent et que nous devons gérer librement les ressources naturelles et la biodiversité qui s'y trouvent, en les conservant comme nous l'avons fait depuis des siècles beaucoup mieux que les occidentaux (et même si nous ne les conservons pas, c'est notre problème "). Ces leaders ajoutent en général à cet argument les justifications 1 à $4^{13}$ ( version indigène de la double conservation).

Enfin, on rencontre trois "versions instrumentales" de la double conservation

$\rightarrow$ Les financeurs de la coopération internationale, comme ceux de la Banque Mondiale à partir de la fin des années 1990, disent :- 8: «Les populations indigènes sont les plus pauvres du pays et elles doivent connaître un traitement de faveur dans nos nouvelles politiques centrées sur les stratégies focalisées de lutte contre la pauvreté ». Ils reprenent également souvent les justifications 1 et 2, particulièrement la 4 sur les cercles vicieux entre destruction environnementale et culturelle (version lutte contre la pauvreté focalisée).

$\rightarrow$ Les économistes, eux, insisteraient plutôt sur la justification suivante:- 9 - « La diversité naturelle et la diversité culturelle doivent être préservées car elles sont des capitaux à mobiliser pour augmenter la richesse générale ou, sinon, à transférer à travers le marché mondial vers d'autres acteurs mieux à même de les rendre productifs ». Cela rejoint la tendance lourde qui consiste à valoriser par la privatisation et le marché le patrimoine culturel et naturel (version économiciste de la double conservation).

$\rightarrow$ Les militants de l'altermondialisation font également de plus en plus usage du discours de la double conservation à travers leur objectif - 10 -: « les destructions environnementales, l'oppression des minorités culturelles, et, peut-être plus emblématique encore, la marchandisation des savoirs traditionnels sur la biodiversité, constituent les meilleures preuves de l'uniformisation inique et des dérèglements provoqués par la globalisation néolibérale que nous combattons. ". Suivant le degré de sophistication, cet argument peut être combiné, en plus de 1 et 2 avec ceux de la version forte $3+4+5$, (version militants altermondialiste).

Cette "carte de navigation" permet d'avoir une vision synthétique des divergences, de mieux se repérer dans la nébuleuse des discours qui portent sur le lien entre peuples autochtones et conservation de la nature. Comme toute rhétorique internationale qui a réussi, l'idée qu'il existe une relation forte entre politique indigéniste et politique de conservation de la nature, entre diversité culturelle et diversité biologique, repose sur un accord minimal. Cette apparence de consensus a facilité sa large diffusion mais a aussi largement occulté les conflits entre les acteurs.

\section{En guise de conclusion}

L'étude du croisement entre politiques de conservation de la nature et de défense des peuples autochtones montre que l'instrumentalisation a été réciproque. Cependant, cet échange de légitimité s'est fait dans des conditions de forte inégalité et n'a été irremplaçable que pour l'internationalisation des droits des autochtones, domaine qui peine à se constituer par lui même en véritable régime international. Au niveau institutionnel, la grande période de croissance a été 1994-2000. La lune de miel semble à présent terminée, les "acteurs mixtes" se sont raréfiés, et les impératifs du multiculturalisme et de la conservation de la nature ont 
toujours des difficultés à s'harmoniser. En revanche, le discours sur l'interdépendance entre diversité biologique et culturelle, et ce qu'on appelle ici "l'impératif de la double conservation" se sont répandus et dilués, lorsqu'ils ont été repris pour justifier une multitude de projets politiques, en dépit des points de vue de plus en plus mesurés des experts de chacun des deux champs. Il convient donc de savoir en distinguer plus précisément les différentes versions, comme cela est proposé ci-dessus. Repris par la critique "post-développementaliste" et "alter-mondialiste", par des intellectuels du Mexique, du Brésil, d'Inde ou même en d'Europe, cet impératif est venu nourrir aussi un nouveau courant utopique à la recherche d'une modernité alternative fondée sur la diversité biologique et culturelle.

${ }^{1}$ Ce texte s'inspire du cas de l'Amérique latine. Les informations présentées ci-dessous et les multiples racourcis que je me permets sont basés sur une multitude de lectures et d'entretiens au Mexique, en France, à Genève et Washington. L'analyse devait retracer l'histoire des différents fils qui rendent possibles le succès de tous ces discours "écolo-indigènes" et leur traductions institutionnelles, mais elle ne pouvait se passer non plus d'évoquer le fonctionnement de chacun des nouveaux champs internationaux, avec leurs nombreux projets et acteurs spécialisés.

${ }^{2}$ Voir en particulier. Darell A. POSEY et Graham DUTFIELD, Le marché mondial de la propriété intellectuelle. Droits des communautés traditionnelles et indigènes, Centre de recherches pour le développement International et WWFSuisse, Ottawa/Genève, 1997, et la somme sur « la double conservation» : Darell A. POSEY (ed.), Cultural and Spiritual Values of Biodiversity. A Complementary Contribution to the Global Biodiversity Assessment, Nairobi, UNEP/IntermediateTechnology Publications, 1997. Stephen BRUSH et Doreen STABINSKY (eds.), Valuing Local Knowledge: Indigenous People and Intellectual Property Rights, Washington DC, Island Press, 1996

${ }^{3}$ Cette remarque concerne: les conséquences spécifiques de la transnationalisation sur le rôle de représentation des dirigeants, les phénomènes de clientélisme qui les lient aux ONG du Nord et aux OIG, les distorsions créées par la volonté de créer une "identité autochtone" au niveau mondial, les inégalités au sein même des organisations indigènes et en particulier la domination nord-américaine. Sur ces points, David Dumoulin, Les politiques de conservation de la nature confrontées aux politiques du renouveau indien, une étude transnationale depuis le Mexique, Paris, thèse de doctorat de Sciences Po Paris sous la direction de Guy Hermet, nov. 2003.

${ }^{4}$ Celle en particulier qui est publiée dans le National Geographic en 1992 Mac CHAPIN, « Indigenous Peoples and the Environment in Central America. Research and Exploration », National Geographic, 8 (2) spring, 1992, pp. 232-233 (carte).

${ }^{5}$ Les bases de données sur les savoirs locaux se sont multipliées. Les principales : www.nufic.nl/ciran/ikdm (NUFFIC-CIRAN correspond à un réseau de plus de 25 centres de recherche dans le monde (avec son bulletin Indigenous Knowledge and Developemnt Monitor).

${ }^{6}$ IPBN pour son sigle en anglais, dont le programme "Indigenous Knowledge" a pris beaucoup d'importance.

${ }^{7}$ Deux facettes du programme de la double conservation: dimension territoriale et démarcation pour la première, et lien entre langues menacées et biodiversité de l'autre. La dirigeante de l'organisation Tierra Lingua, Luisa MAFFI, est une des experts-militants importants dans la diffusion du programme de la double conservation. Site Web www.tierralingua.org; Luisa MAFFI, On Biocultural Diversity : Linking Language and Environment, Washington DC, Smithonian Institution Press, 2001

${ }^{8}$ Ces trois textes datant de 1989,1991 et 1993 contiennent en effet de nombreuses références à l'usage tradtionnel des ressources naturelles, au lien au territoire ou à la dimension spirituelle de leur lien à l'environnement naturel. Leurs définitions des peuples indigènes n'échappent guère à une certaine naturalisation, ou au moins à une perception figée.

${ }^{9}$ UICN: Résol. 1.49-1.56 du Congrès Mondial de 1996, ainsi que les Guidelines spécifiques au WWF .

${ }^{10}$ Les experts-militants de la thématique évitent une vision statique et fermée de la communauté, mais défendent plutôt le renforcement de son autonomie politique, qui passe par la démocratie interne et l'ouverture vers le marché mondial La reprise de leurs travaux peut certes provoquer des dérives: sur la "métaphysique territoriale, cf Tania MURRAY LI, «Purification ethnique, savoir récursif et dilemmes du territorialisme», Revue Internationale des Sciences Sociales, 173, sept. 2002. pp. 401-412.

${ }^{11}$ Tous ces débats débordent largement à présent l'ancienne formulation, centrée sur les connaissances "indigènes", et l'engouement officiel pour la médecine traditionnelle. Par exemple, on a pu voir un ancien membre du Programme Peoples \& Plants de 1'UNESCO créer, en 2001, «La Fondation pour la Diversité Globale, pour la Promotion de la diversité culturelle, biologique et agricole dans le monde» ou le très sérieux « The Amazon Conservation Team » travailler avec les organisations amérindiennes pour "développer un nouveau modèle de conservation bioculturelle "Voir les multiples activités et les contacts de ces deux entités www.globaldiversity.org.uk, www.ethnobotany.org.

${ }^{12}$ L'idée de fonction réservoir pour l'autochtonie date des premières critiques des anthropologues dissidents des années 1970, qui valorisaient les ethnies marginalisées comme des réservoirs d'alternatives de civilisation (Cf. travaux de Darcy Ribeiro sur le processus de civilisation).

${ }^{13}$ Le modèle de l'Etat-Nation est peut être menacé, à travers un phénomène de "privatisation collective" des ressources au nom d'un multiculturalisme réparateur. En fonction de la radicalité de leurs revendications sur l'autonomie des territoires indigènes, ces dirigeants prennent plus ou moins en compte les intérêts "de l'humanité", et savent souvent utiliser l'argument 5 sur la justification normative universaliste de la double conservation. 